\title{
Efeito de Thidiazuron na frutificação da macieira 'Daiane' em condições adversas a polinização
}

\author{
${ }^{1}$ Vera Lucia Scapin Vieira, ${ }^{2}$ José Luiz Petri, ${ }^{3}$ Gentil Carneiro Gabardo, \\ ${ }^{4}$ Caroline de Fátima Esperança, ${ }^{5}$ André Amarildo Sezerino, ${ }^{6}$ Bianca Schveitzer
}

${ }^{1}$ Universidade do Alto Vale do Rio do Peixe - UNIARP, Caçador/SC CEP: 89500-000, Caçador, SC, Brasil.

Email: veraluciacdr@hotmail.com

${ }^{2}$ Empresa de pesquisa Agropecuária e Extensão Rural de Santa Catarina - EPAGRI, Rua Abilio Franco, 1500. CxP 591

CEP 89500-00Caçador-SC. e-mail: petri@epagri.sc.gov.br

${ }^{3}$ Universidade do Estado de Santa Catarina, Centro de Ciências Agroveterinárias, Lages-SC, Brasil. Email: ge.gabardo@gmail.com ${ }^{4}$ Universidade do Alto Vale do Rio do Peixe - UNIARP, Caçador/SC CEP: 89500-000, Caçador, SC, Brasil.

Email: carol.esperanca@hotmail.com

${ }^{5}$ Empresa de Pesquisa Agropecuária e Extensão Rural de Santa Catarina - EPAGRI, Rua Abilio Franco, 1500. CxP 591

CEP 89500-00Caçador-SC. email: andresezerino@epagri.sc.gov.br

${ }^{6}$ Empresa de Pesquisa Agropecuária e Extensão Rural de Santa Catarina - EPAGRI, Rua Abilio Franco, 1500. CxP 591

CEP 89500-00Caçador-SC. email: biancaschveitzer@epagri.sc.gov.br

ISSN 2448-0479

Resumo - O objetivo desse estudo foi avaliar concentraçóes crescentes de Thidiazuron (TDZ), durante dois ciclos produtivos, sobre o efeito na frutificação efetiva, produção e possíveis alterações nas características dos frutos de macieiras da cv. Daiane. Os experimentos foram conduzidos em pomar experimental no município de Caçador, SC. O delineamento experimental utilizado foi o de blocos casualisados com seis tratamentos e cinco repetiçóes. Em arranjo fatorial $2 \times 6$ (ciclo produtivo $\mathrm{x}$ doses de TDZ), sendo os seguintes tratamentos: Controle (sem aplicação); TDZ (5ppm, 10ppm, 15ppm, 20ppm e 25ppm), nos ciclos 2012/2013 e 2013/2014. Em os ciclos avaliados, os tratamentos foram aplicados no estádio de plena floraçáo (F2). Avaliou-se a produção por planta, massa fresca média de frutos $(\mathrm{g})$, frutificação efetiva (\%), coloração vermelha dos frutos (\%), resistência da polpa (lib pol-2), sólidos solúveis totais e índice de iodo-amido. Em média a frutificação efetiva das plantas tratadas com TDZ foi superior às plantas não tratadas. Os tratamentos com aplicaçóes de TDZ mostraram efeito aditivo sobre as variáveis de produção, sendo que os melhores resultados foram observados nas concentraçóes a partir de $10 \mathrm{ppm}$. A aplicação de TDZ pode reduzir a massa média dos frutos, além de afetar as características físico-químicas e reduzir a porcentagem de cobertura vermelha da epiderme dos frutos. A aplicação do Thidiazuron aumenta a produção por planta com concentraçôes a partir de 10ppm, promovendo maior fixação de frutos e consequente- mente aumentando a produção. No entanto, diminui a coloração vermelha dos frutos na macieira 'Daiane', com maior intensidade nas concentrações maiores.

Palavras-chave - Frutificação efetiva. Coloração dos frutos. Malus domestica Borkh.

Abstract - The aim of this study was to evaluate increasing concentrations of Thidiazuron (TDZ) for two production cycles, on the effect on fruit set, production and possible changes in the characteristics of the apple fruit cv. Daiane. The experiments were conducted in an experimental orchard in Caçador municipality, SC, Brazil. The experimental design was randomized blocks with six treatments and five replications. Factorial arrangement $2 \times 6$ (production cycle $\mathrm{x}$ TDZ doses), with the following treatments: control (no application); TDZ (5ppm, 10ppm, $15 \mathrm{ppm}, 20 \mathrm{ppm}$ and $25 \mathrm{ppm}$ ), the cycles 2012/2013 and 2013/2014. In the evaluated cycles, treatments were applied in full bloom stage (F2). We evaluated the production per plant, average fresh mass of fruit (g), fruit set (\%), red color of fruits (\%), pulp strength (lib pol- $\left.{ }^{2}\right)$, total soluble solids and iodine-starch content. On average effective fruiting of plants treated with TDZ was superior to the untreated plants. Treatments with TDZ applications showed additive effect on production variables, the best results were obtained at concentrations from $10 \mathrm{ppm}$. The application of TDZ can reduce the average fruit weight, and affect the physicochemical characteristics and 
reduce the percentage of coverage of the red skin of the fruit. The application of Thidiazuron increases the production per plant with concentrations from $10 \mathrm{ppm}$, promoting greater fruit set and hence increasing production. However, decreases the red colouration of fruit in apple 'Daiane', with greater intensity at the highest concentrations.

Keywords - Fruit set. fruit color. Malus domestica Borkh.

Recebido em: 22 de junho de 2016.

Aprovado em: 24 de novembro de 2016.

\section{INTRODUÇÁO}

Mesmo na região Sul do Brasil, mesmo em regióes propicias ao cultivo da macieira, algumas variações climáticas anuais influenciam no desenvolvimento da planta e polinização, podendo inclusive limitar o cultivo em algumas áreas de menor altitude e maior temperatura, pois as necessidades de frio da planta não são satisfeitas (HAWERROTH et al. 2009).

Uma série de anomalias podem ser observadas em regiões de inverno ameno onde o requerimento em frio das plantas não é totalmente satisfeito, destacando-se a brotação irregular e o florescimento desuniforme e insuficiente. Quanto mais tarde ocorrer a floração, maior a possibilidade de ocorrer temperaturas mais elevadas que podem influenciar negativamente no período de viabilidade dos óvulos (LEITE et al., 2010), promovendo a diminuiçấo da frutificação efetiva.

A frutificação efetiva, como resultado de polinizaçấo bem-sucedida, é requisito para se obter alto rendimento na produçáo das fruteiras de clima temperado (GUERRA et al., 2009). Devido à autoincompatibilidade gametofítica, a maioria das cultivares de macieiras, para viabilizar a polinização cruzada, necessita do plantio de cultivares polinizadoras que apresentem, além de compatibilidade, período de floraçáo coincidente com a cultivar de interesse econômico, indicando-se uma percentagem de plantas polinizadoras de no mínimo $10 \%$ do total das plantas (PETRI, 2006). Uma vez que a polinização anemófila tem pouca ou nenhuma expressão, a transferência do pólen deve ser intermediada por insetos polinizadores (FAORO, 2009).

Contudo, em diversas áreas do mundo as populaçôes desses insetos estão sendo reduzidas a níveis abaixo dos quais podem sustentar serviços de poli- nização adequados em agroecossistemas (KEVAN; VIANA, 2003; FOOD AND AGRICULTURE ORGANIZATION OF THE UNITED NATIONS, 2004). O fenômeno conhecido como "Colony Collapse Disorder" provocou o desaparecimento da população de abelhas melíferas (Apis mellifera) nos Estados Unidos a partir do ano de 2007 e se expandiu pelo mundo, acarretando prejuízos no setor produtivo (COX-FOSTER et al., 2007; DE JONG, 2010; SEZERINO; ORTH, 2015).

Além disso, diversos fatores podem levar a baixa frutificação na macieira, como: o arranjo das polinizadoras inadequado; deficiência nutricional; variaçôes climáticas, pluviometria, umidade relativa do ar, vento, geadas ou baixas temperaturas durante ou após a floração; alternância produtiva induzida por alta produção no ciclo anterior; produtos fitossanitários; má formação das estruturas florais; aborto de embriôes; desbalanço nutricional e hormonal; efeito de porta-enxertos (MELO, 2011).

Devido a esses vários fatores desfavoráveis a frutificaçáo efetiva, o uso de reguladores de crescimento tem sido utilizado na fruticultura como ferramenta para a melhoria da produtividade e qualidade dos frutos, atuando como mediadores de processos metabólicos e fisiológicos. Dentre os reguladores de crescimento destaca-se o Thidiazuron (TDZ), o qual é uma feniluréia com ação citocinínica que estimula a divisão celular, e consequentemente aumenta a frutificação efetiva, no entanto, influencia o crescimento, a forma e o amadurecimento dos frutos em algumas espécies (PETRI et al., 2001).

A maioria das informaçôes disponíveis sobre o uso de TDZ são referentes as macieiras Gala e seus clones, pouco se sabe sobre seus efeitos na cultivar Daiane. A cultivar 'Daiane' é fruto do trabalho do programa de melhoramento da macieira da Epagri, lançada no ano de 1998, tendo como característica principal a resistência a mancha de Glomerela e a colheita no período entre 'gala' e 'Fuji' (DENARDI et al., 2015). Diante disso, o objetivo do trabalho foi avaliar concentraçôes crescentes de TDZ, durante dois anos, sobre o efeito na frutificaçấo efetiva e produção de macieiras da cv. Daiane, bem como, possíveis alteraçóes nas características dos frutos.

\section{MATERIAIS E MÉTODOS/METODOLOGIA}

Os experimentos foram conduzidos em pomar experimental no município de Caçador, SC (latitude 
2650' S, longitude 5058' O, altitude 960 metros), nos ciclos 2012/2013 e 2013/2014. Segundo classificação de Köppen, o clima na regiáo de cultivo é classificado como $\mathrm{Cfb}$ - temperado constantemente úmido, com verão ameno. A média da precipitação pluvial anual é de $1653,2 \mathrm{~mm}$ e a umidade relativa do ar média é de $77,9 \%$.

Utilizaram-se plantas de 12 anos da cv. Daiane, enxertadas sobre o porta-enxerto M-7, tendo como polinizadora a cv. Sansa. A densidade de plantio no pomar utilizado é de 1.000 plantas ha ${ }^{-1}$, com espaçamento de $5 \mathrm{~m}$ entre linhas e $2 \mathrm{~m}$ entre plantas, sendo as plantas manejadas no sistema de condução em líder central. Desde a implantação do experimento até o término da realização deste estudo, o pomar foi conduzido de acordo com as práticas de manejo recomendadas no sistema de produção da macieira (SANHUEZA et al., 2006).

O delineamento experimental utilizado foi o de blocos casualisados com seis tratamentos e cinco repetiçôes. Em arranjo fatorial 2 × 6 (ciclo produtivo $\mathrm{x}$ doses de TDZ), sendo os seguintes tratamentos: 1) Controle (sem aplicação de TDZ); 2) TDZ 5ppm; 3) TDZ 10ppm; 4) TDZ 15ppm; 5) TDZ 20ppm; 6) TDZ 25ppm; nos ciclos 2012/2013 e 2013/2014. Em ambos os ciclos avaliados, os tratamentos foram aplicados no estágio de plena floração (F2). Na safra de 2012/2013, realizou-se a aplicação em 09/10/2012 e na safra de 2013/2014 em 15/10/2013.

Avaliou-se a produção por planta ( $\mathrm{kg}$ planta ${ }^{-1}$ e frutos planta $\left.{ }^{-1}\right)$, massa fresca média de frutos (g), frutificação efetiva ([número de frutos/número de inflorescências] x 100), coloração vermelha dos frutos (\%), resistência da polpa (lib pol-2), sólidos solúveis totais ( ${ }^{\circ}$ Brix) e índice de iodo-amido (1-9).

Os dados de frutificação efetiva, produção $(\mathrm{kg}$ e frutos planta $^{-1}$ ) e índice de iodo/ amido transformados em log $(\mathrm{x})$ e; massa média dos frutos transformados em $\log (x+2)$. Os dados de porcentagem de cobertura vermelha dos frutos foram analisados em delineamento inteiramente casualizado, sendo os dados referentes à porcentagem de frutos com mais de $80 \%$ da epiderme vermelha transformados em raiz (x). Quando verificada significância, procedeuse à comparação de médias pelo teste de Scott-Knott a $5 \%$ de probabilidade de erro. As análises estatísticas foram executadas com o programa Sisvar v.5.6 (FERREIRA, 2010).

\section{RESULTADOS E DISCUSSÃO}

A frutificação efetiva mostrou-se variável entre os anos de estudo, sendo que no primeiro ciclo, $2012 / 2013$, foi relativamente alta nas plantas não tratadas com TDZ, quando comparada a frutificação efetiva do ciclo seguinte, 2013/2014 (Tabela 1). No primeiro ciclo de avaliação não se observaram diferenças entre plantas tratadas e não tratadas, indiferentemente das concentraçôes de TDZ aplicadas.

Tabela 1 - Frutificação efetiva (\%), em plantas de macieira 'Daiane' submetida a diferentes concentraçóes de Thidiazuron (TDZ) nas safras 2012/2013 e 2013/2014. Caçador - SC, 2016.

\begin{tabular}{cccc}
\hline \multirow{2}{*}{$\begin{array}{c}\text { Concentração } \\
\text { Thidiazuron (ppm) }\end{array}$} & \multicolumn{3}{c}{ Frutificaçáo efetiva (\%) } \\
\cline { 2 - 4 } & $\mathbf{2 0 1 2 / 2 0 1 3}$ & $\mathbf{2 0 1 3 / 2 0 1 4}$ & Média \\
\hline $\mathbf{0}$ & $25,3 \mathrm{Aa}$ & $9,3 \mathrm{Bb}$ & $17,3 \mathrm{~B}$ \\
$\mathbf{5}$ & $39,4 \mathrm{Aa}$ & $49,1 \mathrm{Aa}$ & $44,3 \mathrm{~A}$ \\
$\mathbf{1 0}$ & $39,7 \mathrm{Ab}$ & $95,1 \mathrm{Aa}$ & $67,4 \mathrm{~A}$ \\
$\mathbf{1 5}$ & $32,6 \mathrm{Ab}$ & $84,9 \mathrm{Aa}$ & $58,8 \mathrm{~A}$ \\
$\mathbf{2 0}$ & $29,8 \mathrm{Ab}$ & $80,4 \mathrm{Aa}$ & $55,1 \mathrm{~A}$ \\
$\mathbf{2 5}$ & $38,9 \mathrm{Ab}$ & $98,6 \mathrm{Aa}$ & $68,8 \mathrm{~A}$ \\
\hline Média & $34,3 \mathrm{~b}$ & $69,6 \mathrm{a}$ & 51,9 \\
\hline
\end{tabular}

Médias seguidas de mesma letra maiúscula na coluna e minúscula na linha não diferem-se entre si, pelo teste de Scott Knott a 5\% de probabilidade. $C V=12,24 \%$.

Já no ciclo seguinte, a frutificação efetiva nas plantas náo tratadas mostrou-se bastante inferior as do ciclo anterior e também a das plantas tratadas com TDZ, que foi significativamente aumentada, indiferente da concentraçáo de TDZ aplicada. Segundo Warmund (2007), a falta de coincidência do período de floração entre as polinizadoras e a cultivar comercial pode refletir em polinização deficiente, que gera baixa frutificação efetiva ou baixa produção. Petri (2006) aponta os fatores climáticos como limitantes a frutificação da macieira, principalmente o fator temperatura, que pode afetar consideravelmente as taxas de autopolinização ou polinização cruzada entre locais ou anos.

Em média a frutificação efetiva das plantas tratadas com TDZ foi superior a das plantas não tratadas. Esses efeitos sobre a frutificação efetiva, também são relatados por Petri et al. (2010) que observou aumento na frutificação efetiva e o número de frutos por planta da cultivar Royal Gala tratada com TDZ.

Leite et al. (2010) relataram que o número de sementes por fruto não foi influenciado pela aplicação dos reguladores de crescimento TDZ e PCa (Prohexadine 
Cálcio), porem chamam a atenção para o reduzido número de sementes presentes nos frutos, que pode ser atribuído a fatores restritivos a polinização no pomar ou o aumento do abortamento de embrióes e sementes que pode ser estimulado pelo TDZ. No presente trabalho observou-se reduzido número de sementes por frutos em ambos os ciclos (Tabela 2), no entanto não houve diferença na formação de sementes nos frutos de plantas tratadas e não tratadas, o que também foi relatado por Amarante et al. (2002) em macieiras da cultivar Gala. A baixa formação de sementes por frutos aqui observada, pode ser atribuída a fatores ambientais, ou principalmente a baixa densidade de plantas polinizadoras na área de estudo e irregularidades na floraçâo destas. Segundo Petri et al. (2008) altos índices de frutificação efetiva são obtidos quando há coincidência no florescimento entre cultivares polinizadoras e produtoras.

Tabela 2 - Número médio de sementes por fruto de frutos de plantas de macieira 'Daiane' submetida a diferentes concentrações de Thidiazuron (TDZ) nas safras 2012/2013 e 2013/2014. Caçador - SC, 2016.

\begin{tabular}{cccc}
\hline \multirow{2}{*}{$\begin{array}{c}\text { Concentraçáo } \\
\text { Thidiazuron (ppm) }\end{array}$} & \multicolumn{3}{c}{ Sementes fruto $^{-1}$} \\
\cline { 2 - 4 } & $\mathbf{2 0 1 2 / 2 0 1 3}$ & $\mathbf{2 0 1 3 / 2 0 1 4}$ & Média \\
\hline $\mathbf{0}$ & $2,33^{\mathrm{ns}}$ & 2,52 & $2,42 \mathrm{~A}$ \\
$\mathbf{5}$ & 1,74 & 2,18 & $1,96 \mathrm{~B}$ \\
$\mathbf{1 0}$ & 2,42 & 2,78 & $2,60 \mathrm{~A}$ \\
$\mathbf{1 5}$ & 1,83 & 2,88 & $2,35 \mathrm{~A}$ \\
$\mathbf{2 0}$ & 1,70 & 1,96 & $1,83 \mathrm{~B}$ \\
$\mathbf{2 5}$ & 2,35 & 2,94 & $2,64 \mathrm{~A}$ \\
\hline Média & $2,06 \mathrm{~b}$ & $2,54 \mathrm{a}$ & 2,30 \\
\hline
\end{tabular}

Médias seguidas de mesma letra maiúscula na coluna e minúscula na linha não diferem-se entre si, pelo teste de Scott Knott a $5 \%$ de probabilidade. $^{\text {ns }}=$ Não significativo. $\mathrm{CV}=24,78 \%$.

A produção por planta (kg.planta ${ }^{-1} \mathrm{e}$ frutos.plan$\mathrm{ta}^{-1}$ ) foi expressivamente menor no ciclo 2012/2013 do que no ciclo 2013/2014, não sendo constatado nenhum efeito aditivo na produção das plantas tratadas com TDZ, indiferentemente da concentração aplicada (Tabelas 3 e 4). Porém no segundo ciclo, 2013/2014, houve incremento significativo na produção das plantas tratadas com TDZ em comparação as plantas não tratadas, com destaque para as plantas tratadas com a concentração de $10 \mathrm{ppm}$ de TDZ, que expressaram produção por planta $(\mathrm{kg}) 188,2 \%$ superior as plantas não tratadas. Em média, os tratamentos com aplicações de TDZ mostraram efeito aditivo sobre as variáveis de produção analisadas neste estudo, sendo que os melhores resultados foram observados nas concentraçôes a partir de 10ppm. Estes resultados corroboram com os resultados obtidos por Petri et al. (2001), onde foi observado que Thidiazuron aplicado a 10ppm aumentou a frutificação efetiva e o tamanho das frutas da macieira cv. Gala.

Tabela 3 - Produção (kg.planta ${ }^{-1}$ ), em plantas de macieira 'Daiane' submetida a diferentes concentraçóes de Thidiazuron (TDZ) nas safras 2012/2013 e 2013/2014. Caçador - SC, 2016.

\begin{tabular}{cccc}
\hline \multirow{2}{*}{$\begin{array}{c}\text { Concentraçáo } \\
\text { Thidiazuron (ppm) }\end{array}$} & \multicolumn{3}{c}{ Produçáo (kg.planta-1) } \\
\cline { 2 - 4 } & $\mathbf{2 0 1 2 / 2 0 1 3}$ & $\mathbf{2 0 1 3 / 2 0 1 4}$ & Média \\
\hline $\mathbf{0}$ & $14,6 \mathrm{Ab}$ & $30,6 \mathrm{Da}$ & $22,6 \mathrm{C}$ \\
$\mathbf{5}$ & $16,5 \mathrm{Ab}$ & $51,8 \mathrm{Ca}$ & $34,2 \mathrm{~B}$ \\
$\mathbf{1 0}$ & $24,3 \mathrm{Ab}$ & $88,2 \mathrm{Aa}$ & $56,2 \mathrm{~A}$ \\
$\mathbf{1 5}$ & $23,3 \mathrm{Ab}$ & $68,9 \mathrm{Ba}$ & $46,1 \mathrm{~A}$ \\
$\mathbf{2 0}$ & $25,8 \mathrm{Ab}$ & $66,3 \mathrm{Ba}$ & $46,0 \mathrm{~A}$ \\
$\mathbf{2 5}$ & $30,4 \mathrm{Ab}$ & $66,4 \mathrm{Ba}$ & $48,4 \mathrm{~A}$ \\
\hline Média & $22,6 \mathrm{~b}$ & $62,0 \mathrm{a}$ & 42,2 \\
\hline
\end{tabular}

Médias seguidas de mesma letra maiúscula na coluna e minúscula na linha nâo diferem-se entre si, pelo teste de Scott Knott a 5\% de probabilidade. $\mathrm{CV}=23,73 \%$.

Tabela 4 - Produção (frutos.planta ${ }^{-1}$ ), em plantas de macieira 'Daiane' submetida a diferentes concentraçóes de Thidiazuron (TDZ) nas safras 2012/2013 e 2013/2014. Caçador - SC, 2016.

\begin{tabular}{cccc}
\hline \multirow{2}{*}{$\begin{array}{c}\text { Concentração } \\
\text { Thidiazuron } \\
\text { (ppm) }\end{array}$} & \multicolumn{3}{c}{ Produçáo (frutos.planta ${ }^{-1}$ ) } \\
\cline { 2 - 4 } & $\mathbf{2 0 1 2 / 2 0 1 3}$ & $\mathbf{2 0 1 3 / 2 0 1 4}$ & Média \\
\hline $\mathbf{0}$ & $96,0^{\text {ns }}$ & $161,4 \mathrm{C}$ & $128,7 \mathrm{C}$ \\
$\mathbf{5}$ & 125,6 & $324,2 \mathrm{~B}$ & $224,9 \mathrm{~B}$ \\
$\mathbf{1 0}$ & 190,2 & $654,2 \mathrm{~A}$ & $422,2 \mathrm{~A}$ \\
$\mathbf{1 5}$ & 166,2 & $474,2 \mathrm{~B}$ & $320,2 \mathrm{~A}$ \\
$\mathbf{2 0}$ & 194,0 & $426,0 \mathrm{~B}$ & $310,0 \mathrm{~A}$ \\
$\mathbf{2 5}$ & 216,4 & $467,8 \mathrm{~B}$ & $342,1 \mathrm{~A}$ \\
\hline Média & $164,7 \mathrm{~b}$ & $417,9 \mathrm{a}$ & 291,3 \\
\hline
\end{tabular}

Médias seguidas de mesma letra maiúscula na coluna e minúscula na linha náo diferem-se entre si, pelo teste de Scott Knott a 5\% de probabilidade. ${ }^{\text {ns }}=$ Náo significativo. CV $=4,61 \%$.

Segundo Leite et al. (2010) a resposta da aplicação dos reguladores de crescimento, como o TDZ, está mais relacionada com a época de aplicação do que com a concentraçáo aplicada, sendo que os melhores resultados foram observados no período inicial do desenvolvimento dos frutos, queda de pétalas. Alguns autores como Bianchi et al. (2000) e Petri et al. (2001) advertem quanto ao risco de deformaçóes nos frutos pelo uso de concentraçóes altas de TDZ.

Da mesma forma que foi visto nas variáveis relacionadas a produção, a massa média dos frutos também sofreu alteração entre os ciclos estudados, com destaque para o ciclo 2013/2014 que expressou os 
maiores valores (Tabela 5). Ao analisar a média dos dois ciclos, torna-se visível o efeito da aplicação de TDZ sobre a massa média dos frutos, que em geral é reduzida quando comparada as plantas náo tratadas. Esta redução na massa média dos frutos esta correlacionada com o aumento da produção por planta promovida pela aplicação do TDZ (FAGUNDES, 2015).

Tabela 5 - Massa média (g.fruto ${ }^{-1}$ ), em plantas de macieira 'Daiane' submetida a diferentes concentraçôes de Thidiazuron (TDZ) nas safras 2012/2013 e 2013/2014. Caçador - SC, 2016.

\begin{tabular}{cccc}
\hline \multirow{2}{*}{$\begin{array}{c}\text { Concentraçáo } \\
\text { Thidiazuron (ppm) }\end{array}$} & \multicolumn{4}{c}{ Massa (g.fruto- $\left.^{-1}\right)$} \\
\cline { 2 - 4 } & $\mathbf{2 0 1 2 / 2 0 1 3}$ & $\mathbf{2 0 1 3 / 2 0 1 4}$ & Média \\
\hline $\mathbf{0}$ & $150,8 \mathrm{Ab}$ & $190,9 \mathrm{Aa}$ & $170,8 \mathrm{~A}$ \\
$\mathbf{5}$ & $131,2 \mathrm{Bb}$ & $160,2 \mathrm{Ba}$ & $145,7 \mathrm{~B}$ \\
$\mathbf{1 0}$ & $128,2 \mathrm{Ba}$ & $136,4 \mathrm{Ca}$ & $132,3 \mathrm{C}$ \\
$\mathbf{1 5}$ & $140,9 \mathrm{Aa}$ & $146,3 \mathrm{Ca}$ & $143,6 \mathrm{~B}$ \\
$\mathbf{2 0}$ & $133,2 \mathrm{Bb}$ & $153,9 \mathrm{Ba}$ & $143,5 \mathrm{~B}$ \\
$\mathbf{2 5}$ & $140,7 \mathrm{Aa}$ & $142,0 \mathrm{Ca}$ & $141,4 \mathrm{~B}$ \\
\hline Média & $137,5 \mathrm{~b}$ & $154,9 \mathrm{a}$ & 146,2 \\
\hline
\end{tabular}

Médias seguidas de mesma letra maiúscula na coluna e minúscula na linha não diferem-se entre si, pelo teste de Scott Knott a 5\% de probabilidade. $\mathrm{CV}=0,64 \%$.

Alguns aspectos físico químicos dos frutos no momento da colheita podem sofrer alteraçóes em virtude da aplicação de TDZ e também das condiçôes climáticas de cada ciclo produtivo (Tabela 6). No primeiro ciclo desse estudo, a firmeza da polpa dos frutos não sofreu alteração entre os tratamentos, sendo que os valores de firmeza foram altos em comparaçáo ao ciclo seguinte. Já no ciclo 2013/2014, bem como nos dados médios dos ciclos estudados, o TDZ aumentou a firmeza de polpa dos frutos, indiferentemente da concentração aplicada.

Tabela 6 - Firmeza da polpa (lb.pol-2) em plantas de macieira 'Daiane' submetida a diferentes concentrações de Thidiazuron (TDZ) nas safras 2012/2013 e 2013/2014. Caçador - SC, 2016.

\begin{tabular}{cccc}
\hline \multirow{2}{*}{$\begin{array}{c}\text { Concentraçáo } \\
\text { Thidiazuron (ppm) }\end{array}$} & \multicolumn{3}{c}{ Firmeza da polpa (lb.pol $\left.{ }^{-2}\right)$} \\
\cline { 2 - 4 } & $\mathbf{2 0 1 2 / 2 0 1 3}$ & $\mathbf{2 0 1 3 / 2 0 1 4}$ & Média \\
\hline $\mathbf{0}$ & $17,6 \mathrm{Aa}$ & $14,1 \mathrm{Bb}$ & $15,8 \mathrm{~B}$ \\
$\mathbf{5}$ & $17,8 \mathrm{Aa}$ & $16,0 \mathrm{Ab}$ & $16,9 \mathrm{~A}$ \\
$\mathbf{1 0}$ & $18,1 \mathrm{Aa}$ & $15,3 \mathrm{~A} \mathrm{~b}$ & $16,7 \mathrm{~A}$ \\
$\mathbf{1 5}$ & $17,8 \mathrm{Aa}$ & $16,3 \mathrm{Ab}$ & $17,0 \mathrm{~A}$ \\
$\mathbf{2 0}$ & $18,3 \mathrm{Aa}$ & $16,9 \mathrm{Ab}$ & $17,6 \mathrm{~A}$ \\
$\mathbf{2 5}$ & $17,3 \mathrm{Aa}$ & $16,0 \mathrm{Ab}$ & $16,6 \mathrm{~A}$ \\
\hline Média & $17,8 \mathrm{a}$ & $15,7 \mathrm{~b}$ & 16,8 \\
\hline
\end{tabular}

Médias seguidas de mesma letra maiúscula na coluna e minúscula na linha não diferem-se entre si, pelo teste de Scott Knott a 5\% de probabilidade. $\mathrm{CV}=4,83 \%$.
Segundo Fagundes (2015) a aplicação de TDZ pode promover atraso na maturação dos frutos, visto que pode reduzir os teores de sólidos solúveis, aumentar a firmeza de polpa dos frutos em comparação as plantas não tratadas. No entanto o índice de maturação dos frutos, dado pela relação iodo/amido, não foi alterado pelos tratamentos, no entanto, entre os ciclos foi observada diferença, sendo que o índice de maturação dos frutos foi maior no segundo ciclo de estudo (Tabela 7).

Tabela 7 - Iodo/Amido de frutos de plantas de macieira 'Daiane' submetida a diferentes concentraçóes de Thidiazuron (TDZ) nas safras 2012/2013 e 2013/2014. Caçador - SC, 2016.

\begin{tabular}{cccc}
\hline \multirow{2}{*}{$\begin{array}{c}\text { Concentraçáo } \\
\text { Thidiazuron } \\
(\mathbf{p p m})\end{array}$} & \multicolumn{3}{c}{ Iodo/amido (1-9) } \\
\cline { 2 - 4 } & $\mathbf{2 0 1 2 / 2 0 1 3}$ & $\mathbf{2 0 1 3 / 2 0 1 4}$ & Média \\
\hline $\mathbf{0}$ & $3,4^{\mathrm{ns}}$ & 8,1 & $5,7^{\mathrm{ns}}$ \\
$\mathbf{5}$ & 3,2 & 8,8 & 6,0 \\
$\mathbf{1 0}$ & 2,6 & 8,3 & 5,4 \\
$\mathbf{1 5}$ & 3,5 & 8,4 & 5,9 \\
$\mathbf{2 0}$ & 2,9 & 7,3 & 5,1 \\
$\mathbf{2 5}$ & 3,7 & 8,0 & 5,8 \\
\hline Média & $3,2 \mathrm{~b}$ & $8,1 \mathrm{a}$ & 5,7 \\
\hline
\end{tabular}

Médias seguidas de mesma letra maiúscula na coluna e minúscula na linha não diferem-se entre si, pelo teste de Scott Knott a 5\% de probabilidade. $^{\text {ns }}=$ Náo significativo. $\mathrm{CV}=10,91 \%$.

A aplicação de TDZ promoveu redução nos teores de sólidos solúveis totais (SST\%), porem houve variação entre as concentrações aplicadas e os ciclos estudados (Tabela 8). Ao observarmos a média entre os ciclos avaliados fica evidenciado a redução nos SST\% promovida pela aplicação do TDZ.

Tabela 8 - Sólidos Solúveis Totais (SST) de frutos de plantas de macieira 'Daiane' submetida a diferentes concentraçōes de Thidiazuron (TDZ) nas safras 2012/2013 e 2013/2014. Caçador - SC, 2016.

\begin{tabular}{cccc}
\hline \multirow{2}{*}{$\begin{array}{c}\text { Concentraçáo } \\
\text { Thidiazuron } \\
(\mathbf{p p m})\end{array}$} & \multicolumn{3}{c}{ SST $\left({ }^{\circ} \mathrm{Brix}\right)$} \\
\cline { 2 - 4 } & $\mathbf{2 0 1 2 / 2 0 1 3}$ & $\mathbf{2 0 1 3 / 2 0 1 4}$ & Média \\
\hline $\mathbf{0}$ & $13,3 \mathrm{Aa}$ & $13,6 \mathrm{Aa}$ & $13,4 \mathrm{~A}$ \\
$\mathbf{5}$ & $13,0 \mathrm{Aa}$ & $12,7 \mathrm{Ba}$ & $12,8 \mathrm{~B}$ \\
$\mathbf{1 0}$ & $12,8 \mathrm{Ba}$ & $12,1 \mathrm{Cb}$ & $12,5 \mathrm{~B}$ \\
$\mathbf{1 5}$ & $12,6 \mathrm{Ba}$ & $12,6 \mathrm{Ba}$ & $12,6 \mathrm{~B}$ \\
$\mathbf{2 0}$ & $12,7 \mathrm{Ba}$ & $12,0 \mathrm{Cb}$ & $12,4 \mathrm{~B}$ \\
$\mathbf{2 5}$ & $12,5 \mathrm{Ba}$ & $12,5 \mathrm{Ba}$ & $12,5 \mathrm{~B}$ \\
\hline Média & $12,8 \mathrm{a}$ & $12,6 \mathrm{~b}$ & 12,7 \\
\hline
\end{tabular}

Médias seguidas de mesma letra maiúscula na coluna e minúscula na linha não diferem-se entre si, pelo teste de Scott Knott a 5\% de probabilidade. $\mathrm{CV}=2,81 \%$. 
Em ambos os ciclos avaliados, a porcentagem de cobertura vermelha da epiderme dos frutos sofreu alteração em virtude da aplicação do TDZ (Tabela 9). Naturalmente, sem aplicação de TDZ, os frutos têm maior distribuição na categoria de frutos com cobertura de coloração vermelha da epiderme superior a $80 \%$ (>80). Porem com a aplicaçấo de TDZ, indiferentemente da concentração, ocorreu uma drástica redução da porcentagem de frutos na categoria de frutos mais coloridos (>80) e significativo aumento nas categorias de menor coloração (<50 e 50-80). De acordo com Fagundes (2015), a porcentagem de coloração vermelha dos frutos está diretamente relacionada com a firmeza de polpa, os sólidos solúveis totais, o índice iodo/amido, o comprimento e o diâmetro de frutos.

Tabela 9 - Porcentagem média de frutos em cada categoria de acordo com a cobertura vermelha (\%) de plantas de macieira 'Daiane' submetida a diferentes concentraçóes de Thidiazuron (TDZ) na safra 2012/2013. Caçador - SC, 2016.

\begin{tabular}{|c|c|c|c|c|c|c|}
\hline \multirow{3}{*}{$\begin{array}{c}\text { TDZ } \\
(\text { ppm) }\end{array}$} & \multicolumn{6}{|c|}{ Cobertura vermelha da epiderme (\%) } \\
\hline & \multicolumn{3}{|c|}{$2012 / 2013$} & \multicolumn{3}{|c|}{$2013 / 2014$} \\
\hline & $<50$ & $50-80$ & $>80^{*}$ & $<50$ & $50-80$ & $>80^{*}$ \\
\hline 0 & $6,0 \mathrm{~b}$ & & & $10,8 \mathrm{c}$ & & $63,8 \mathrm{a}$ \\
\hline 5 & $17,6 \mathrm{a}$ & & & $30,6 \mathrm{a}$ & & $27,8 \mathrm{~b}$ \\
\hline 10 & $11,6 b$ & $43,6 a$ & $44,8 b$ & $22,6 b$ & $42,0 \mathrm{a}$ & $35,4 b$ \\
\hline 15 & $14,3 \mathrm{a}$ & $39,0 \mathrm{a}$ & $46,7 b$ & $45,0 \mathrm{a}$ & $38,0 \mathrm{a}$ & $17,0 \mathrm{c}$ \\
\hline 20 & $10,3 b$ & $48,0 \mathrm{a}$ & & $35,6 \mathrm{a}$ & & $23,2 \mathrm{c}$ \\
\hline 25 & $17,2 \mathrm{a}$ & $47,5 \mathrm{a}$ & $35,3 b$ & $25,6 b$ & $37,0 \mathrm{a}$ & $37,4 \mathrm{~b}$ \\
\hline $\mathrm{CV}(\%)$ & 40,6 & 21,0 & 10,0 & 35,1 & 19,3 & 19,1 \\
\hline
\end{tabular}

Médias seguidas de mesma letra na coluna, não diferem-se entre si, pelo teste de Scott Knott a 5\% de probabilidade. *Dados originais transformados em raiz quadrada.

\section{CONCLUSÁO}

A aplicação do Thidiazuron aumenta a produção por planta com concentraçóes a partir de 10ppm, promovendo maior fixaçáo de frutos e consequentemente aumentando a produção. A aplicação de TDZ diminui a coloração vermelha dos frutos na macieira 'Daiane', com maior intensidade nas concentraçôes maiores.

\section{AGRADECIMENTOS}

Agradecemos a Empresa de Pesquisa Agropecuária e Extensão Rural de Santa Catarina - EPAGRI, pelo apoio e infraestrutura para realização deste trabalho.

\section{REFERÊNCIAS}

AMARANTE, C.V.T. et al. Efeito do thidiazuron no crescimento, no florescimento, na frutificação e na nutrição em macieiras. Pesquisa Agropecuária Brasileira, Brasília, v. 37, n.10, p. 1365-1372, 2002.

BIANCHI, V. J. et al. Aumento da frutificação efetiva em pereiras cultivar garber com uso de AG3 e TDZ. Revista Brasileira de Agrociência, v.6, n.3, p.191-193, 2000.

COX-FOSTER, D. L. et al. A metagenomic survey of microbes in honey bee Colony Collapse Disorder. Science, Washington, v. 318, n. 5848, p. 283-287, 2007.

DEJONG, D. Desaparecimento das abelhas - Brasil. 2010. Disponível em: < https://www.yumpu.com/pt/document/ view/12819758/desaparecimento-das-abelhas-brasil-dejong-d-depto-de- >. Acesso em: 3 maio 2013.

DENARDI, F.; KVITSCHAL, M.V.; HAWERROTH, M.C. SCS425 Luiza: Novo cultivar de macieira com sabor de polpa de alto padrão. Florianópolis: Epagri, 2015. Disponível em: < https://www.embrapa.br/web/mobile/ publicacoes/-/publicacao/1045260/scs-luiza-novo-cultivar-de-macieira-com-sabor-de-polpa-de-alto-padrao >. Acesso em 06/05/2016.

FAGUNGES, E. Efeito da concentraçáo e época de aplicação de tidiazurom na frutificaçáo e qualidade de frutos da macieira 'Royal Gala'. 2015. 76f. Dissertação (Mestrado) - Programa de Pós-graduação em Produção Vegetal, Centro de Ciências Agroveterinárias, Universidade do Estado de Santa Catarina, Lages, 2015.

FAORO, I. D. Biologia reprodutiva da pereira japonesa (Pyrus pyrifolia var. Culta) sob o efeito do genótipo e do ambiente. 2009. 219 f. Tese (Doutorado em Recursos Genéticos Vegetais) - Faculdade de Agronomia, Universidade Federal de Santa Catarina, Florianópolis, 2009 .

FERREIRA, D. F. SISVAR - programa estatístico. Versão 5.3 (Build 75). Lavras: Universidade Federal de Lavras, 2010 .

FOOD AND AGRICULTURE ORGANIZATION OF THE UNITED NATIONS. Conservation and management of pollinators for sustainable agriculture - the international response. In: FREITAS, B. M.; PEREIRA, J. O. P. (Ed.). Solitary bees: conservation, rearing and management for pollination. Fortaleza: Imprensa Universitária, 2004, p. 19-25.

GUERRA, M. E. et al. S-RNase genotyping and incom- 
patibility group assignment by PCR and pollination experiments in Japanese plum. Plant Breeding, Berlin, v.128, n.3, p. 304-311, 2009.

HAWERROTH, F. J et al. Fenologia, brotação de gemas e produção de frutos de macieira em resposta à aplicação de cianamida hidrogenada e óleo mineral. Bragantia, Campinas, v.68, n.4, p.961-971, 2009.

KEVAN, P.G.; VIANA, B.F. The global decline of pollination services. Biodiversity, v.4, n. 4, p. 3-8, 2003.

LEITE, G. B. et al. Increasing apple fruit set on "Condessa" using growth regulators. Acta Horticulturae, Leuven, n. 884, p. 537-544, 2010.

MELO, G.M.S. Uso de reguladores de crescimento como alternativa tecnológica na cultura da macieira 'Eva' (Malus domestica Borkh). 2011. 67 f. Dissertação (Mestrado) - Faculdade de Ciências Agronômicas de Botucatu, Universidade Estadual Paulista, Botucatu.

PETRI, J. L., SCHUCK, E, LEITE, G. B. Efeito do Thidiazuron (TDZ) na Frutificação de Fruteiras de Clima Temperado. Revista Brasileira de Fruticultura, Jaboticabal, v. 23, n. 32001.

PETRI, J. L.; HAWERROTH, F. J.; LEITE, G. B. Fenologia de espécies silvestres de macieira como polinizadora das cultivares Gala e Fuji. Revista Brasileira de Fruticultura, Jaboticabal, v. 30, n. 4, p. 868-874, 2008.

PETRI, J. L. et al. Effect of growth regulators on 'Gala' apple fructification. Acta Horticulturae, Leuven, n. 884, p. 531-536, 2010.

PETRI, J.L. Fatores edafoclimáticos. In: EMPRESA DE PESQUISA AGROPECUÁRIA E EXTENSĀO RURAL DE SANTA CATARINA. A cultura da macieira. Florianópolis: EPAGRI, 2006. p.105-111.

SANHUEZA, R.M.V.; PROTAS, J.F.S.; FREIRE, J.M. Manejo da Macieira no Sistema de Produçáo Integrada de Frutas. Bento Gonçalves: Embrapa Uva e Vinho, 2006.

SEZERINO, A. A.; ORTH, A. I. Limitações ao uso de Apis mellifera (Hymenoptera: Apidae) para a polinização dirigida de cultivares: um estudo de caso com a pereira-portuguesa (Pyrus communis L. cv. Rocha). Biotemas, Florianópolis, v. 28, n. 2, p. 73-86, 2015.

WARMUND, M.R. Pollinating fruit crops. Columbia: University of Missouri. Disponível em: <http://extension. missouri.edu/ explorepdf/agguides/hort>. Acesso em: 20 maio 2016. 